\title{
Performance Evaluation of Live Virtual Machine Migration in SDN-enabled Cloud Data Centers ${ }^{\text {th }}$
}

\author{
TianZhang $\mathrm{He}^{\mathrm{a}, *}$, Adel Nadjaran Toosi ${ }^{\mathrm{b}}$, Rajkumar Buyya ${ }^{\mathrm{a}}$ \\ ${ }^{a}$ Clouds Computing and Distributed Systems (CLOUDS) Laboratory, School of Computing \\ and Information Systems, The University of Melbourne Parkville, VIC 3010, Australia \\ ${ }^{b}$ Information Technology, Monash University Clayton, VIC 3800, Australia
}

\footnotetext{
Article history: Received 4 July 2018, Received in revised from 1 February 2019, Accepted 17 April 2019, Available online 26 April 2019

TianZhang He, Adel N. Toosi, Rajkumar Buyya, Performance evaluation of live virtual machine migration in SDN-enabled cloud data centers, Journal of Parallel and Distributed Computing, Volume 131, 2019, Pages 55-68, ISSN 0743-7315, https://doi.org/10.1016/j.jpdc.2019.04.014.

${ }^{*}$ Corresponding author.

Email addresses: tianzhangh@student.unimelb.edu.au (TianZhang He), adel.n.toosi@monash.edu (Adel Nadjaran Toosi), rbuyya@unimelb.edu.au (Rajkumar Buyya)
} 


\begin{abstract}
In Software-Defined Networking (SDN) enabled cloud data centers, live VM migration is a key technology to facilitate the resource management and fault tolerance. Despite many research focus on the network-aware live migration of VMs in cloud computing, some parameters that affect live migration performance are neglected to a large extent. Furthermore, while SDN provides more traffic routing flexibility, the latencies within the SDN directly affect the live migration performance. In this paper, we pinpoint the parameters from both system and network aspects affecting the performance of live migration in the environment with OpenStack platform, such as the static adjustment algorithm of live migration, the performance comparison between the parallel and the sequential migration, and the impact of SDN dynamic flow scheduling update rate on TCP/IP protocol. From the QoS view, we evaluate the pattern of client and server response time during the pre-copy, hybrid post-copy, and auto-convergence based migration.
\end{abstract}

Keywords: Live VM migration, Software-Defined Networking, Cloud Computing, Virtual Machine, Performance Measures, OpenStack, OpenDaylight

2010 MSC: 00-01, 99-00 


\section{Introduction}

With the rapid adoption of cloud computing environments for hosting a variety of applications such as Web, Virtual Reality, scientific computing, and big data, the need for delivering cloud services with Quality of Service (QoS) guar5 antees is becoming critical. For cloud data center management, it is important to prevent the violation of Service Level Agreement (SLA) and maintain the QoS in heterogeneous environments with different application contexts. Therefore, there has been a lot of focus on optimizing the service latency and energy efficiency dynamically in order to benefit both cloud computing tenants and providers. Virtual Machines (VMs), as one of the major virtualization technologies to host cloud services, can share computing and networking resources. In order to alleviate SLA violation and meet the QoS guarantees, the placement of VMs needs to be optimized constantly in the dynamic environment. Live VM migration is the key technology to relocate running VMs between physical hosts without disrupting the VMs' availability [1]. Thus, in SDN-enabled data centers, live VM migration as a dynamic management tool facilities various objectives of the resource scheduling [2, 3, 4, such as load balancing, cloud bursting, resource overbooking, and energy-saving strategy, fault tolerance, scheduled maintenance as well as evacuating VMs to other data centers before the incidents like earthquake and flooding which require VM location adjustment.

The live VM migration technologies can be categorized into the precopy memory [1] and post-copy memory migration [5]. During the precopy live migration, the Virtual Machine Monitor (VMM), such as KVM and Xen, iteratively copy memory (dirty pages produced in the last round) from the running VM at the source host to the VM container at the target host. However, the post-copy live migration first suspends the VM at the source host and resumes the VM at the target host by migrating a minimal subset of VM execution state. At the same time, the source VM still pro-actively pushing the remained pages to the resumed VM. A page-fault happens when the VM attempt to access an un-transferred page which can be solved by fetching the 
pages from the source VM. However, in many circumstances such as in Wide Area Network (WAN) environment (inter-data centers and between edge and core cloud computing), and some production Data Centers where some servers don't share the same storage system, there is no Network File System (NFS) 35 between the source and the target hosts to share ephemeral disks. The live migration with block storage, also called block live migration, is used by combining memory migration with live disk migration. Besides the memory migration of live VM migration, the live disk migration is used [6, 7] to transfer the ephemeral disk of the VM instance to the target host.

The goal of this paper is to tackle an important aspect in the field of VM migration, namely understanding the parameters that affect the performance of live VM migration in SDN-enabled cloud computing. The performance of live migration could be evaluated by measuring three metrics:

- Migration Time is the time duration from the initialization of the premigration process on the source host to the successful completion of the post-migration process on both hosts.

- Downtime refers to the duration that the VM is suspended due to the stop-and-copy, Commitment and Activation phase. From the client perspective, the service is unavailable.

- Transferred Data is the amount of data transferred between the source and destination host during the migration process.

There are continuous efforts to improve live VM migration, such as improving the performance of live migration algorithm [8, 9, modeling for better prediction of the cost [10, 11, network-aware live migration to alleviate the influence of migration on SLA and application QoS [12, 13, 14, optimizing the multiple live VM migration planning [15, 16, 17, 18, and benchmarking the live migration effects on applications [19, 20]. Nonetheless, many parameters, such as downtime adjustment and non-network overheads, that affect the live migration time and downtime are neglected to a large extent. During a live VM 
migration, the downtime threshold for the last memory-copy iteration could be changed as time elapses. This will affect the memory-copy iteration rounds, which leads to different migration time and downtime. Computing overheads of live VM migration can also constitute a large portion of total migration time, which will affect the performance of multiple VM migrations.

On the other hand, some work focus on the live VM migration in SoftwareDefined Networking (SDN) scenarios [15, 2, 18]. By virtualizing the network resources, we could use SDN to dynamically allocate bandwidth to services and control the route of network flows. Due to the centralized controller, SDN can provide a global view of the network topology, states of switches, and statistics on the links (bandwidth and latency). Based on the information, orchestrator can calculate the 'best' path for each flow and call SDN controller Northbound APIs to push the forwarding rules to each switch in the path. However, the latencies of the flow entry installation on the switch and the communication between SDN controller and switches could impact the traffic engineering performance in the SDN-enabled cloud data centers. Thus, the scheduling update rate of choosing the 'best' path will affect the live migration traffic.

Moreover, although some work [19, 20, focus on the impacts of live migration on the cloud services, such as multi-tier web application, the worst-case response time pattern as well as the technologies, such as hybrid post-copy and autoso convergence, for a successful live migration need to be investigated further. Hybrid post-copy (H-PC) [5] is the strategy that combines pre-copy and postcopy migration. The post-copy mode will be activated after the certain precopy phase where most of the memory has been transferred. Based on the CPU throttling, Auto-convergence (AC) 21] will decrease the workload where the memory write speed is relative to the CPU executing speed.

We evaluate the live migration time, downtime, and total transferred data using OpenStack [22] as the cloud computing platform. OpenStack uses the pre-copy live migration with the default driver Libvirt (virtualization API) [23]. Our study is fundamentally useful to resource scheduling, such as energy-saving 90 strategy, load balancing, and fault tolerant, driven by SLA. The contributions 
are fourfold, and are summarized as follows:

- Evaluation of the performance of block live migration in OpenStack with different configuration of static downtime adjustment algorithm. Experimental results can be used as reference to dynamically configure optimal migration time and downtime.

- Modeling and identification of the trade-off between sequential and parallel migration when the host evacuation happens in the same network path.

- Evaluation of the effect of flow scheduling update rate on the migration performance as well as TCP/IP protocol in SDN-enabled clouds. Experimental results can guide to optimize the update rate and select the best path of SDN forwarding scheduler in order to achieve better migration performance.

- Evaluation of the response time of a multi-tier web application under precopy, hybrid post-copy and auto-convergence based live migration. Specifically, experimental results demonstrate the worst-case response time and the situation when the pre-copy migration could not finish in a reasonable time.

The rest of the paper is organized as follows. Section 2 introduces the related work and motivations. In Section 3, we present the system overview of SDN-enabled data centers and details of the live migration in OpenStack. The mathematical models of block live migration, sequential and parallel migrations are presented in Section 4. In Section 5, we describe the objectives, testbed specifications, metrics, and the experimental setup of the evaluated parameters. We quantitatively show how these parameters can dramatically affect the migration time and service performance. Finally, we conclude our work in Section 6.

\section{Related Work}


Clark et al. [1] firstly proposed the live VM migration comparing to the naive stop-and-copy method. During the iterative memory copy phase of live migration implemented in Xen virtualization platform, rapid dirtying pages which updated extremely frequently, called Writable Working Set (WWS), was introduced. These pages will not be transmitted to the destination host in the iteration round in order to reduce the total migration time and transferred data. In addition, the authors elaborated the implementation issues and features with regard to the managed migration (migration daemons of Xen in host and destination hosts), self migration (implementation the mechanism within the OS), dynamic rate-limiting for each iteration round, rapid page dirtying, and paravirtualized optimizations (stunning rogue process, i.e. limit write faults of each process, and freeing page cache pages, i.e. reclaiming back cold buffer cache pages). Although pre-copy migration is widely used in various virtualization platforms, such as Xen, QEMU/KVM, VMWare, it is worth noting that migration algorithms and performance of different hypervisors are different in terms of dirty pages detection and transmission and stop-and-copy threshold 24]. For instance, the page skip (WWS) mechanism does not be implemented in KVM.

In order to alleviate the overheads caused by live VM migrations, the prediction model is required to estimate the live migration performance in advance. Akoush et al. [10] proposed a model to estimate the migration time and downtime of live VM migration based on the two main functions of migration, i.e. peek and clean. The peek function returns the dirty bitmap and the clean function returns the dirty pages and resets them to clean state. They used both average dirty page rate (AVG) and history based page dirty rate (HIST) in their prediction algorithms. The HIST model could capture the variability of live migration and help to decide the moment at which migration begins to minimize the migration cost. Moreover, Liu et al. 25] introduced the rapid 145 page dirtying in its migration performance prediction model. In order to obtain a more accurate prediction model, the authors refined the previous prediction model of migration performance by estimating the size of WWS. Based on the observation, it is an approximated proportional size of the total dirty pages in 
each iterative memory copy round with regard to previous iteration time and

dirty pages rate. The authors also proposed an energy consumption model of live migration based on the linear regression between total transferred data and measured energy consumption. The synthesized cost for migration decision is based on the estimated values of downtime, migration time, transferred data, and energy cost. Furthermore, based on prediction model of migration cost, different migration strategies for load balancing, fault toleration, and server consolidation are proposed [1]. The algorithms choose the proper migration candidates in order to minimize the total migration cost while satisfying the requirements of rescheduling algorithms. Contrary to their work we focus on the mechanism and performance of proposed parameters and corresponding models.

Prediction models of live migration which assume a static downtime threshold [10, 25, 11] or constant dirty page rate [18, cannot reflect the real migration time and downtime in OpenStack. The downtime threshold in OpenStack uses a static adjustment algorithm. It is increased monotonically with a certain time interval and steps during the migration in order to reduce the migration time. A misconfigured downtime configuration will lead to a poor performance of live migration, such as unstable downtime which results in the SLA violation and a long-time migration that degrades the network performance. Therefore, in order to dynamically set optimal configurations, we need to have a better understanding of the relationship between downtime adjustment configurations and migration performance in OpenStack.

Planning of the sequential and parallel migration in intra and inter-data centers to optimize the server evacuation time and minimize the influence of live migration has attracted interest recently [15, 16, 18. However, they only focus on the network aspect of multiple live migration planning to decide the sequence of sequential and concurrent live migration in order to minimize the migration duration. As mentioned in [10, the total migration time includes pre-migration, pre-copy phase, stop-and-copy phase and post-migration overheads. The most proportion of migration time could be the operation overheads. Therefore, in 
need to pinpoint the impacts of non-network overheads on the parallel and sequential migration in the same path.

Moreover, Software-Defined Networking (SDN) [26] as a powerful feature in Cloud computing provides a centralized view of topology and bandwidth on every path. We could flexibly implement network scheduling algorithm and set bandwidth limit for live migration and other application traffics. In a highly dynamic network environment, the 'best' path decided by scheduling algorithm based on an update rate could change frequently. Therefore, not only the bandwidth but traffic pattern, SDN control plan 27] and flow table latency 28] could also affect the live migration performance. Understanding the SDN latency in the flow scheduling is very important for achieving better live migration performance.

With different application context, the impacts of live migration on application performance could change dramatically. For instance, the workload of a multi-tier web application with specific write and communication pattern [19, 20] is different with the workload in scientific computing applications. The response time should be soft real-time to satisfy the QoS of application. Therefore, network service suffers more from the disruption due to the downtime and the performance degradation due to the live VM migration. As there are few works on this topic, evaluating the live migration effects on the response time of different types of network-sensitive applications is desirable. However, current work did not consider the worst-case response time and the situation when the pre-copy migration could not finish in a reasonable time. Thus, we need to evaluate the response time distribution of the web application during the migration, and the impacts of strategies, hybrid post-copy, and auto-convergence, on application response time which perform a successful live migration. 


\section{System Overview}

In SDN-enabled data centers, the computing resources are under control of cloud management platform, such as OpenStack, while the networking resources are managed by SDN controller. The management module (orchestrator) coordinates the SDN controller and the OpenStack services by using northbound RESTful APIs to perform VM migration planning in resource scheduling algorithm such as the SLA-aware energy-saving strategy as shown in Fig. 1. In OpenStack, Nova service runs on top of Linux servers as daemons to provides the ability to provision the compute servers. Meanwhile, Neutron component provides 'connectivity as a service' between network interfaces managed by other services like Nova.

More specifically, the cloud controller of Infrastructure as a Service (IaaS) platform, OpenStack, is in charge of configuring and assigning all computing and storage resources, such as allocating flavor (vCPU, memory, storage) to VMs, placing the VMs on physical hosts using Nova component. It keeps all the information about physical hosts and virtual machines, such as residual storage and available computing resources. At the same time, all computer nodes update the states of hosted VMs to OpenStack Nova service. Furthermore, Neutron, the OpenStack network component, provides the management of virtual networking, such as start, update and bind the VM's port, as well as the communication between VMs. However, the OpenStack Neutron does not control network devices (switches). It only controls networking modules in compute nodes and network nodes.

Therefore, the SDN controller uses OpenFlow [29] protocol through southbound interfaces to manage the forwarding planes on network devices (switches). The open-source virtual switch, Open vSwitch (OVS) 30], provides the virtualization switching stack supporting OpenFlow and other standard protocols. Therefore, without expensive dedicated switches, we could install OVS in the white box as the OpenFlow switch in SDN-enabled data centers. Based on the link information between OpenFlow devices, the SDN controller calculates the 


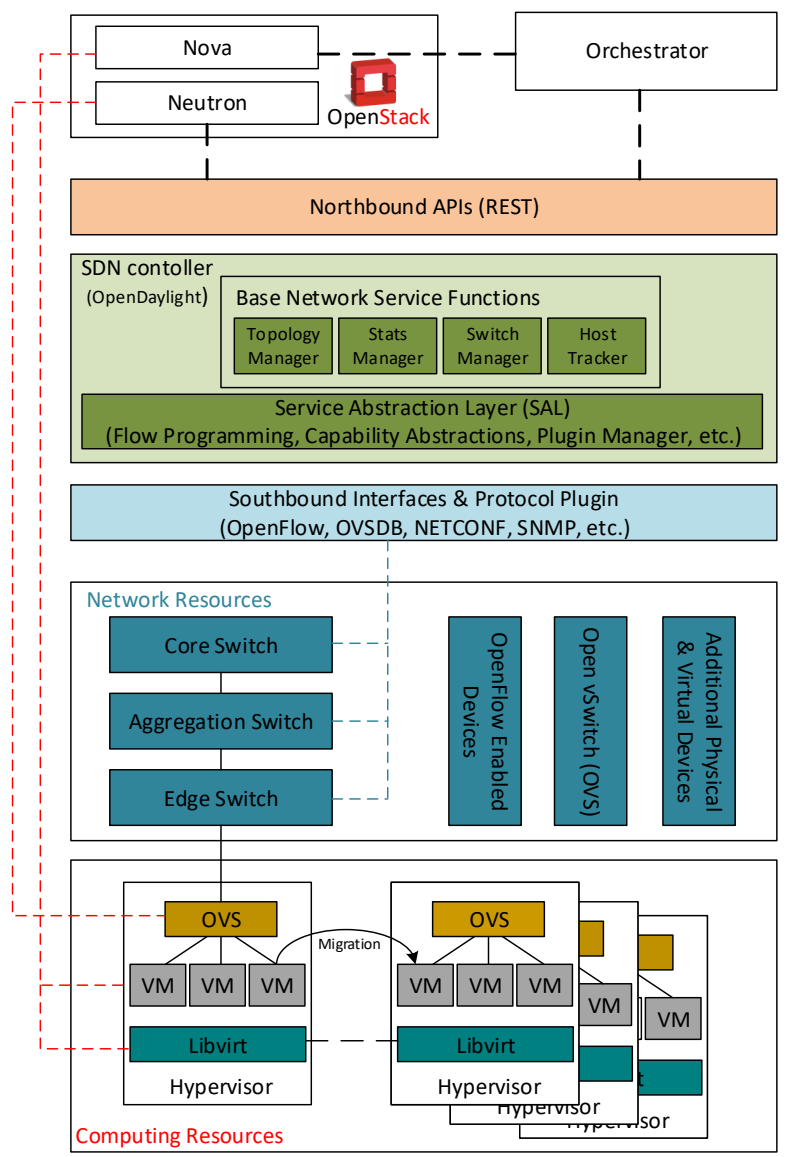

Figure 1: System Overview

forwarding tables for all network traffics. The OpenFlow switches forward the traffic flow according to the received forwarding tables from SDN controller. It also measures the received and transmitted data size as well as the bandwidth and latency between each other.

\subsection{Live Migration in OpenStack}

In this section, we present the details of block live migration in OpenStack. Providing a comprehensive solution to control the computing and network resources in the datacenter, OpenStack uses Libvirt [23] to manage hosts in order 


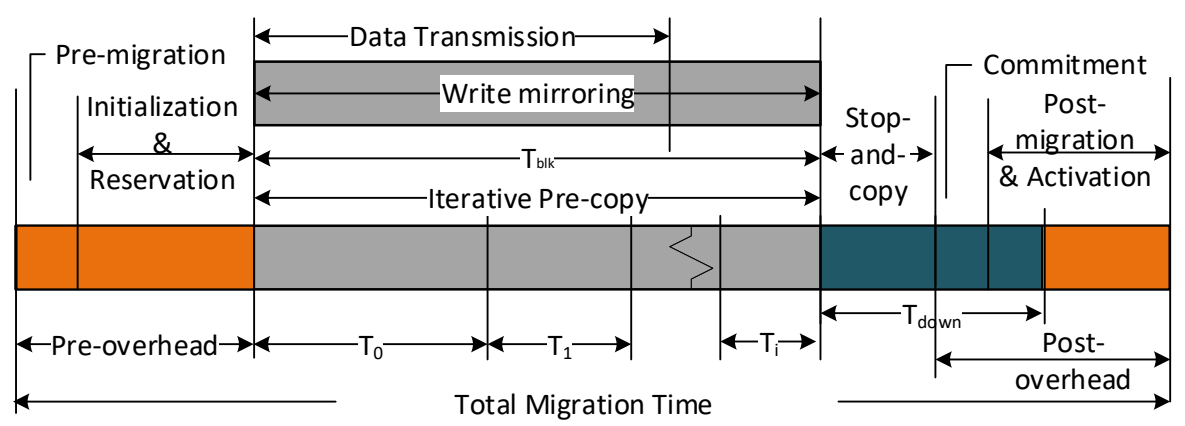

Figure 2: OpenStack block live migration Neutron to perform the pre- and post-live-migration operations, and uses Libvirt to handle the actual live migration operations. The pre-copy live VM migration is used by default driven by libvirt.

Since libvirt 1.0.3, the QEMU's Network Block Device (NBD) server and "drive-mirror" primitive [6] are used to perform live storage migration (without shared storage setup). Similarly, since VMWare ESX 5.0, it uses VMKernel data mover (DM) and IO mirroring to perform live storage migration [7]. It separates the storage streaming data flows from the instance's RAM and hypervisor's internal state data flows. The disk transmission will perform concurrently with three types: 1) Into the block has been migrated, the writes will be mirrored to the target. 2) Into the block being migrated, the writes will be sent to the target first and wait in the queue until the region migration finished. 3) Into the block which will be migrated, the writes are issued to the source disk without mirroring. By caching the backing file or instance image when it boot in the Nova compute host, the mirror action could just apply to the top active overlay in the image chain. Thus, the actual disk transmission will be reduced.

Similar to the pre-copy migration described in [1, the block live migration in OpenStack includes 9 steps (Fig. 2): 
host, updates ports binding, and sets up the logical router with Neutron server.

2. Initialization (Init): Preselects the target host to speed the future migration.

3. Reservation (Reserv): Target host sets up the temporary share file server; and initializes a container for the reserved resource on the target host.

4. Disk Transmission: For live storage migration, starts to perform storage migration and synchronizes the disk through IO mirroring.

5. Iterative pre-copy: For pre-copy VM migration, sends dirty pages that are modified in the previous iteration round to the target host. The entire RAM is sent in the first round.

6. Stop-and-copy: the VM is paused during the last iteration round according to the downtime threshold (remained amount is less than the required).

7. Commitment (Commit): Source host gets the commitment of a successfully received instance copy from the target host.

8. Activation (Act): Reassigns computing resource to the new VM and delete the old VM on the source host.

9. Post-live migration (PostMig): On the target host, updates port state and rebinds the port with Neutron. VIF driver unplugs the VM's port on the source host.

Where copying overheads are due to the pre-copy iteration and downtime is caused by the stop-and-copy, commitment and parts of the activation and postmigration operations. Although the network-related phases (disk transmission, pre-copy iteration, and stop-and-copy) usually dominate the total migration time, the pre- and post-live-migration, initialization, reservation, commitment, 
and activation could add a significant overhead to the migration performance in certain scenarios (large available network bandwidth, small disk size or low dirty page rate). The pre-live-migration, initialization, reservation could be classified as pre-migration overheads while the commitment, activation and post-live-migration as post-migration overheads.

Downtime Adjustment Algorithm: Unlike the stop conditions that are used in QEMU or Xen migration algorithm, the downtime threshold in OpenStack live migration increases monotonically in order to minimize the downtime for lower dirty page rate VM while increasing the availability of high dirty page rate VM migration with a reasonable downtime. The downtime adjustment algorithm used in Libvirt is basically based on three static configuration values (max_downtime, steps, delay):

- live_migration_downtime: The maximum threshold of permitted downtime;

- live_migration_downtime_steps: The total number of adjustment steps until the maximum threshold is reached;

- live_migration_downtime_delay: Multiplies the total data size with the factor equals to the time interval between two adjustment steps in seconds.

For example, the setting tuple $(400,10,30)$ means that there will be 10 steps to increase the downtime threshold with 30 seconds delay for each step up to the 400ms maximum. With the total 3 GB RAM and Disk data size, the downtime threshold at time $\mathrm{t}$, as $T_{d-t h d}(t)$, will be increased at every 90 seconds starting from $40 \mathrm{~ms}$, i.e. $T_{d-t h d}(0)=40 m s, T_{d-t h d}(90)=76 m s, \ldots, T_{d-t h d}(900)=$ $400 \mathrm{~ms}, \ldots, T_{d-t h d}(t>900)=400 \mathrm{~ms}$. The mathematical model of downtime adjustment algorithm is shown in Equation (9). Although OpenStack only support static downtime adjustment in configuration files, we could use the virsh command to interact with the on-going migration based on the elapsed time. 


\section{Mathematical Model}

We present the mathematical model of block live migration as well as the sequential and parallel migrations in the same network path.

\subsection{Block Live Migration}

The mathematical model of block live migration is presented in this section. 325 According to the OpenStack live migration process, the components of pre and post-migration overheads can be represented as:

$$
\begin{aligned}
& T_{\text {pre }}=\text { PreMig }+ \text { Init }+ \text { Reserv } \\
& T_{\text {post }}=\text { Commit }+ \text { Act }+ \text { PostMig }
\end{aligned}
$$

We use $D$ and $M$ to represent the system disk size and the VM memory size, and let $\rho$ denotes the average compression rate used in memory compression algorithm 31]. Let $\rho^{\prime}$ and $R^{\prime}$ denotes the average disk compression rate and mirrored disk write rate. Let $R_{i}$ and $L_{i}$ denote the average dirty page rate need to be copied and bandwidth in iteration round $i$. In total $n$ round iterative pre-copy and stop-and-copy stages, $T_{i}$ denotes the time interval of $i_{t h}$ round iteration shown in Fig. 22 Therefore, the transferred volume $V_{i}$ in round i can be calculated as:

$$
V_{i}=\left\{\begin{array}{cc}
\rho \cdot M & \text { if } i=0 \\
\rho \cdot T_{i-1} \cdot R_{i-1} & \text { otherwise }
\end{array}\right.
$$

As shown in Fig. 2, the time interval of the $i_{t h}$ iteration can be calculated as:

$$
T_{i}=V_{i} / L_{i}=\rho^{i+1} \cdot \prod_{j=0}^{i-1} R_{j} \cdot M / \prod_{j=0}^{i} L_{j}
$$

In [18, they assume that, when $R_{i}, L_{i}$ are constant, the average dirty page rate is not larger than the network bandwidth in every iteration. Let ratio $\sigma=\rho \cdot R / L$. Therefore, $T_{i}=\rho \cdot M \cdot \sigma^{i} / L$. The total time of iterative memory pre-copy $T_{m e m}$ can be calculated as:

$$
T_{m e m}=\frac{\rho \cdot M}{L} \cdot \frac{1-\sigma^{n+1}}{1-\sigma}
$$



as:

$$
T_{b l k} \leq \rho^{\prime} \cdot\left(D+R^{\prime} \cdot T_{b l k}\right) / L
$$

Thus, the upper bound transmission time of the live storage migration is:

$$
T_{b l k} \leq \frac{\rho^{\prime} \cdot D}{L-\rho^{\prime} \cdot R^{\prime}}
$$

For a more accurate $T_{b l k}$, one need to simulate the write behavior based on the actual workload. The network part of block live migration is the maximum 345 value of Equation (4) and (6):

$$
T_{\text {copy }}=\operatorname{Max}\left\{T_{b l k}, T_{m e m}\right\}
$$

The total migration time of block live migration $T_{m i g}$ can be represented as:

$$
T_{m i g}=T_{p r e}+T_{\text {copy }}+T_{\text {post }}
$$

Let $(\theta, s, d)$ denotes the setting tuple (max_downtime, steps, delay) of the downtime adjustment algorithm. Therefore, the live migration downtime threshold at time $t$ can be represented as:

$$
T_{d-t h d}(t)=\lfloor t /(d \cdot(D+M))\rfloor \cdot(\theta s-\theta) / s^{2}+\theta / s
$$

$$
V_{d-t h d}(t)=T_{d-t h d}(t) \cdot L_{n-1}
$$

where $L_{n-1}$ is the n-1 round bandwidth estimated by the live migration algorithm and $L_{n-1}=L$ when transmission bandwidth is a constant.

The live migration changes to the stop-and-copy phase when remained dirty pages is less than the current threshold, as $V_{n} \leq V_{d-t h d}(t)$. Using the Equation (2) in the inequality, the total round of memory iteration can be represented as:

$$
n=\left\lceil\log _{\sigma} \frac{V_{d-t h d}(t)}{M}\right\rceil
$$

Therefore, the upper bound of actual migration downtime is $T_{\text {down }}=T_{d}+$ $T_{\text {post }}^{\prime} \leq T_{d-t h d}(t)+T_{\text {post }}^{\prime}$, where $T_{d}$ is the time that transferring the remained 


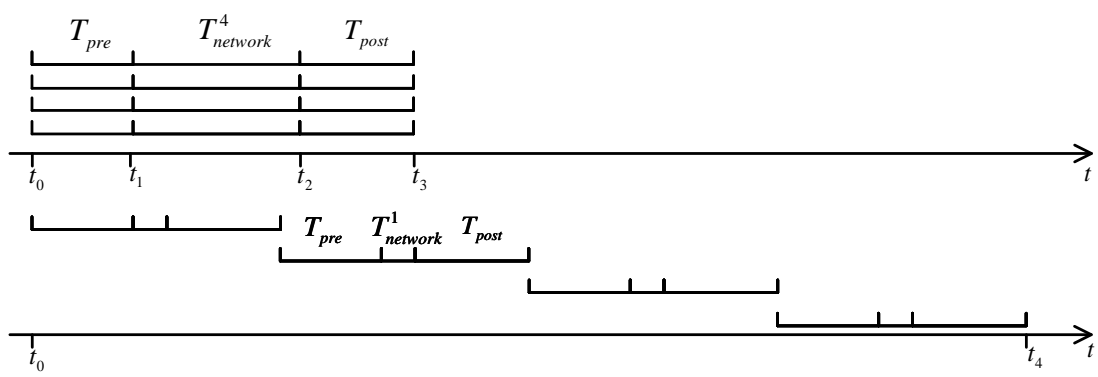

Figure 3: An example of sequential and parallel migrations

dirty pages and storage and $T_{\text {post }}^{\prime}$ is the time spent on the part of post-migration overheads to resume the VM.

360

Because the pre-live-migration process of next migration is executed after the completion of current migration, there is a bandwidth gap between every 
sequential live migration because of the non-network overheads. Therefore, the total evacuation time of $N$ VM sequential migrations could be calculated as the sum of every migration's overhead processing time and network transmission time:

$$
T_{\text {seq }}=\sum_{1}^{N} T_{m i g}=\sum T_{\text {overhead }}+\sum T_{\text {network }}
$$

The response time of VM migration task refers to the time interval from the point that migration task is released and the point it is finished. The migration time indicates the real execution time of the migration task which excludes the waiting time which is the time interval between the migration task release point and the actual start point. The evacuation duration refers to the time interval from the beginning of the first released migration task to the end of the last finished task of all VM migrations.

Pre- and post-migration overheads refer to the operations that are not part of the direct network transmission process. These non-network operations could add a significant overhead to the total migration time and downtime. For more concise explanation, we assume that every VM in parallel migration has same dirty page rate and flavor. Let $m$ denotes the allowed parallel number, $p$ denotes the processing speed of one core. We assume that the largest allowed parallel migration is smaller than the minimum core number on the hosts, $m \leq$ Num(cores), $m \leq N$. When $m>N, m=N$ in the corresponding equations. As every migration sharing the network bandwidth equally, $L / m$ is the transmission rate for each migration. Therefore, using the previous equations, the network transmission time of parallel $m$ migrations can be represented as:

$$
T_{\text {network }}^{m}=\operatorname{Max}\left\{m \cdot T_{b l k}, \frac{m \cdot \rho \cdot M}{L} \cdot \frac{1-(m \sigma)^{n+1}}{1-m \sigma}\right\}
$$

It is clear that $T_{\text {network }}^{m} \geq \Sigma^{m} T_{\text {network }}^{1}$.

Let $W_{\text {pre }}, W_{\text {post }}$ denote the workload of pre and post-migration overheads. As the overheads are significant when the network bandwidth $L$ allocated to the path is more than sufficient or the dirty page rate $R$ is small, we assume that:

$$
\Sigma^{m} W_{\text {pre }} / m \cdot p \geq T_{\text {network }}^{m}
$$


maximum evacuation time of parallel migration $T_{\text {par }}=\operatorname{Max}\left(T_{\text {par }}^{\prime}, T_{\text {par }}^{\prime \prime}\right)$ can be represented as:

$$
\begin{aligned}
T_{\text {par }}^{\prime} & =\frac{\sum_{1}^{X m} W_{\text {pre }}}{m \cdot p}+\frac{\sum_{X m+1}^{N} W_{\text {pre }}}{N-X m+2}+T_{\text {network }}^{N-X}+\frac{\sum_{X m+1}^{N} W_{\text {post }}}{N-X m+2} \\
& =\frac{(\lfloor N / m\rfloor+1) \cdot W_{\text {pre }}+W_{\text {post }}}{p}+T_{n e t w o r k}^{N-X} \\
T_{\text {par }}^{\prime \prime} & =\frac{\sum_{1}^{m} W_{\text {pre }}}{m \cdot p}+T_{\text {network }}^{m}+\frac{\sum_{1}^{X m} W_{\text {post }}}{m \cdot p}+\frac{\sum_{X m+1}^{N} W_{\text {post }}}{N-X m+2} \\
& =\frac{(\lfloor N / m\rfloor+1) \cdot W_{\text {post }}+W_{\text {pre }}}{p}+T_{\text {network }}^{m}
\end{aligned}
$$

As $0 \leq \sigma<1$, we could get the upper bound of parallel network transmission time:

$$
T_{\text {network }}^{m} \leq \operatorname{Max}\left\{m \cdot T_{b l k}, \frac{m \cdot \rho \cdot M}{L \cdot(1-m \sigma)}\right\}
$$

$$
\Delta_{n e t w o r k}=T_{n e t w o r k}^{m}-\Sigma^{m} T_{n e t w o r k}^{1}
$$

$$
\Delta_{\text {workload }}=\Sigma^{m} T_{\text {overhead }}-\Sigma^{m} T_{\text {overhead }} / m \cdot p
$$

Therefore, when $\Delta_{\text {network }}<\Delta_{\text {workload }}$, the evacuation time of parallel migration is smaller than the sequential migration.

All proposed models and results of single migration and sequential and parallel migrations for block live migration also apply to the general live VM migration with disk sharing by deleting the live disk transmission parts, $T_{b l k}$ and $D$, in the models.

\section{Performance Evaluation}

There are several parameters which can influence the live VM migration performance in SDN-enabled data centers from system view, such as the flavor, 
CPU, memory, and static downtime adjustment, network view, such as parallel and sequential migrations, available bandwidth, and dynamic flow scheduling update rate, and application view, such as response time under different migration strategies. In this section, we explore the impacts of these parameters on migration performance. The migration time, downtime, and transferred data shown in the results are the average values. In OpenStack, we can use the nova migration-list to measure the duration of live migration. The downtime of live migration could be calculated by the time stamp difference of VM lifecycle event (VM Paused and VM Resumed) in both Nova log files. Each configured migration experiment is performed 6 times.

Table 1: Specifications of physical hosts in CLOUDS-Pi

\begin{tabular}{cccccc}
\hline Machine & CPU & Cores & Memory & Storage & Nova \\
\hline $3 \times$ IBM X3500 M4 & Xeon(R) E5-2620@ $2.00 \mathrm{GHz}$ & 12 & $64 \mathrm{~GB}(4 \times 16 \mathrm{~GB}$ DDR3 1333MHz) & $2.9 \mathrm{~TB}$ & compute1-3 \\
$4 \times$ IBM X3200 M3 & Xeon(R) X3460 @ $2.80 \mathrm{GHz}$ & 4 & $16 \mathrm{~GB}(4 \times 16 \mathrm{~GB}$ DDR3 1333MHz $)$ & $199 \mathrm{~GB}$ & compute4-7 \\
$2 \times$ Dell OptiPlex 990 & Core(TM) i7-2600@ $3.40 \mathrm{GHz}$ & 4 & $8 \mathrm{~GB}(4 \times 16 \mathrm{~GB}$ DDR3 1333MHz $)$ & $399 \mathrm{~GB}$ & compute8-9 \\
\hline
\end{tabular}

\subsection{Testbed and its Specification}

As current production system will not allow users to access or modify the low-level infrastructure elements, such as resource management interfaces and SDN controllers and switches, needed for experiments, we created our own testbed. CLOUDS-Pi 32], a low-cost testbed environment for SDN-enabled cloud computing, is used as the research platform to test virtual machine block live migration. We use OpenStack combined with OpenDayLight [33] (ODL) SDN controller to manage the SDN-enabled Data Centers, which contains 9 heterogeneous physical machines connected through Raspberry Pis as OpenFlow switches whose specifications are shown in Table 1. The Raspberry Pis are integrated with Open vSwitch (OVS) as 4-port switches with 100 Mbps Ethernet Interfaces. The network physical topology is shown in Fig. 4. The OpenStack version we used is Ocata and the Nova version is 15.0.4 and the Libvirt version is 3.2.0. The Ubuntu tool stress-ng [34] is used as the micro-benchmark 


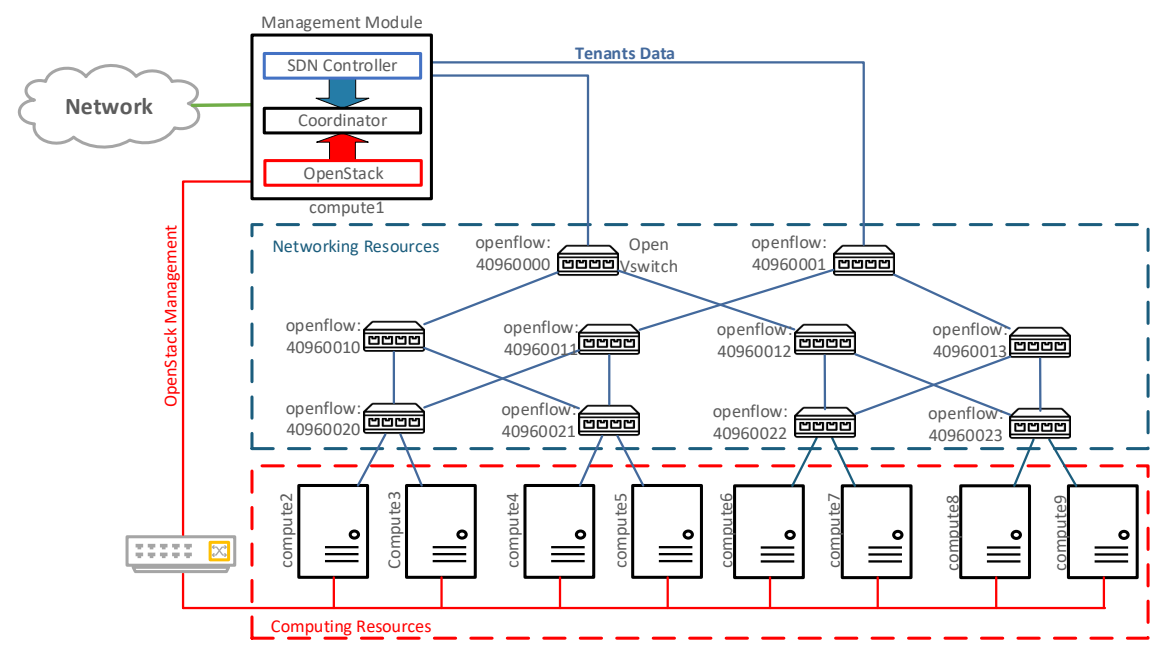

Figure 4: SDN-enabled Data Center Platform

Table 2: Specifications of VM flavors in OpenStack

\begin{tabular}{|c|c|ccc|c|c|ccc|}
\hline No. & Name & vCPUs & RAM & Disk & No. & Name & vCPU & RAM & Disk \\
\hline 1 & nano & 1 & $64 \mathrm{MB}$ & $1 \mathrm{~GB}$ & 5 & medium & 2 & $3.5 \mathrm{~GB}$ & $40 \mathrm{~GB}$ \\
2 & tiny & 1 & $512 \mathrm{MB}$ & $1 \mathrm{~GB}$ & 6 & large & 4 & $7 \mathrm{~GB}$ & $80 \mathrm{~GB}$ \\
3 & micro & 1 & $1 \mathrm{~GB}$ & $10 \mathrm{~GB}$ & 7 & xlarge & 8 & $15.49 \mathrm{~GB}$ & $160 \mathrm{~GB}$ \\
4 & small & 1 & $2 \mathrm{~GB}$ & $20 \mathrm{~GB}$ & & & & & \\
\hline
\end{tabular}

to stress memory and CPU to pinpoint the impacts of parameters on migration performance.

It will allow researchers to test any SDN-related technology in the real environment. Allowed network speed in the testbed is scaled together with the size of computing cluster. Although the testbed's scale is small regarding the number of computer nodes and the network, it can represent the key elements in the large-scale systems. The evaluation results produced by the testbed will be more serious in a large scale environment. Furthermore, as we do not focus on the IO stress on the migrating storage, the evaluation results could also benefit the live migration with shared storage, as well as the live container migration. 

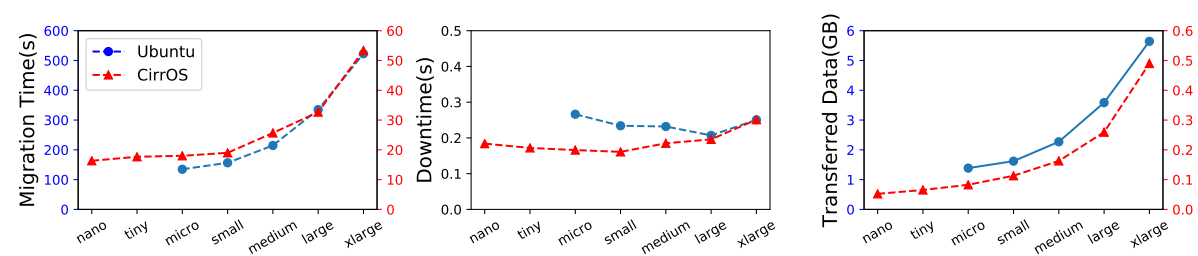

(a) Ubuntu and CirrOS VM with different flavors
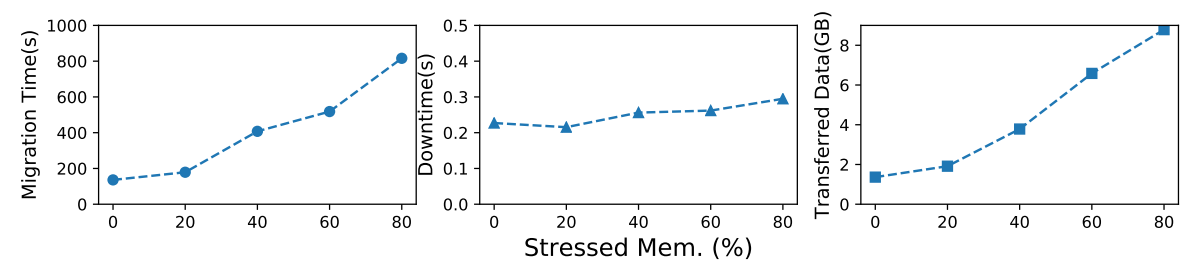

(b) Live migration based on stressed memory
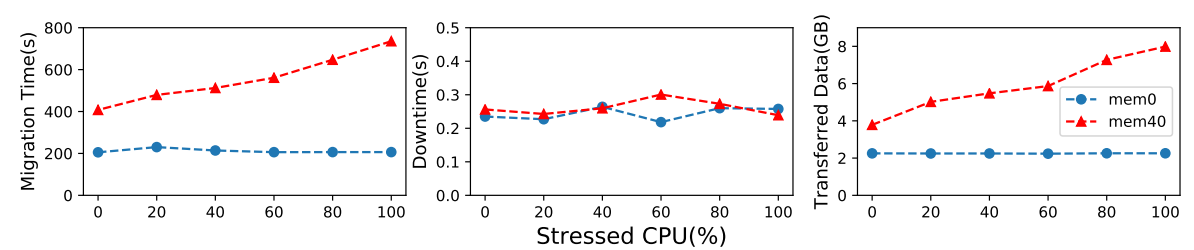

(c) Live migration based on CPU loads

Figure 5: Primary VM parameters

\subsection{Primary Parameters}

First, we evaluate the fundamental parameters, such as flavor, memory and CPU loads, which affect the migration time, downtime and total transferred data of block live VM migration in OpenStack. As we measured, the amount of data from destination to source can be omitted because it only accounts for around 1.8 percent of total transferred data. The transferred data is measured by the SDN controller through OpenFlow protocol. We set 7 flavors in OpenStack, which are nano, tiny, micro, small, medium, large, xlarge (Table 2). Not only the RAM size but the ephemeral disk size can affect the migration time as well as the total transferred data (Equation 8). We evaluate these primary parameters by migrating instances from compute2 to compute3. In the flavor 

est flavor suitable for the Ubuntu image is micro. The image size of CirrOS is 12.65 MB, and Ubuntu is 248.38 MB. In memory stress experiment, we evaluate the migration performance of different memory-stressed Ubuntu-16.04 instance with micro flavor from 0 to 80 percent. In CPU stress memory experiment, 475 we compare the migration performance with 0 to 100 stressed CPU between Ubuntu instance with 0 memory stress (mem0) and 40 percent memory-stressed (mem40) VMs.

Flavor: Figure 5a illustrates the migration performance (migration time, downtime, and total transferred data) of idle VMs with different flavors. Larger The VM block live migration cost with the same flavor could be a huge difference due to the system disk size and the required RAM of different OS instance. According to the downtime adjustment algorithm, a longer migration time can lead to a larger downtime. However, the difference of downtime is small compared to the significant difference of migration time. From flavor micro to xlarge, the transferred data is increased linearly. Furthermore, the transferred date vs. flavor figure illustrates that there is a constant data size difference between CirrOS and Ubuntu with the same flavor. With the same flavor, VM with a larger and more complex OS installed has a longer migration time and larger transferred data as the data size difference of the OS base image and dirty rate caused by OS processes.

Memory: The dirty page rate (and dirty block rate) directly affects the number of pages that are transferred in each pre-copy iteration. Fig. $5 \mathrm{~b}$ shows that the performance of different memory-stressed Ubuntu instances from 0 to 80 percent on the migration time, downtime, total data transferred from source. As shown in Equation (8) and (9), the relationship between the dirty page rate and live migration performance is not linear due to the downtime adjustment algorithm. The downtimes of migrations may be constant with different dirty page rates because of the delay of every downtime adjustment, such as 0 and 20 
downtimes of live migrations with drastically different dirty page rate remain at a stable range.

CPU: Higher CPU workloads can lead to a migration performance degradation because of the page copying operation overhead during the pre-copy iterations. Meanwhile, the high CPU workloads can also cause interference among memory-intensive tasks which leads to a large migration time. We examine the block live migrations based on various CPU loads from 0 to 100 percent. Figure 5c shows that, without stressed memory, the CPU loads inside VMs are irrelevant to the downtime and duration of live migration with the minor copying overhead due to the pre-copy iterations. However, as the CPU usage of a 40-percent-stressed memory task is 100 percent, an extra CPU workload can lead to a larger amount of total transferred data and migration time. For idle VMs, the migration time and transferred data are constant under various range of CPU workload. For more busy VMs, extra CPU workload leads to a linear increase in migration time and transferred data size.

\subsection{Downtime Configuration Effectiveness}

In OpenStack, the live VM migration time could shift dramatically based on different configuration tuples (max_downtime, steps, delay). Although only implemented in OpenStack, the downtime adjustment algorithm can also apply to other cloud computing platforms. In this experiment, the Ubuntu-16.04 instance with micro flavor is migrated between NOVA compute node compute2 and compute3. We perform migrations based on the different step or delay settings and other two default values, i.e., $(500,4,75)$ and $(500,10,5)$, with 0 to 75 percent stressed memory VM.

Figure 6 indicates that for less memory stressed VMs (low dirty page rate), the static algorithm based on short delay could lead to a higher downtime with a slightly different migration time. However, for heavy memory stressed VMs (high dirty page rate), the adjustment of large delay setting, such as delay40, delay110, leads to an extremely long migration duration. The larger adjustment step setting leads to a larger migration time with a smaller downtime. However, 

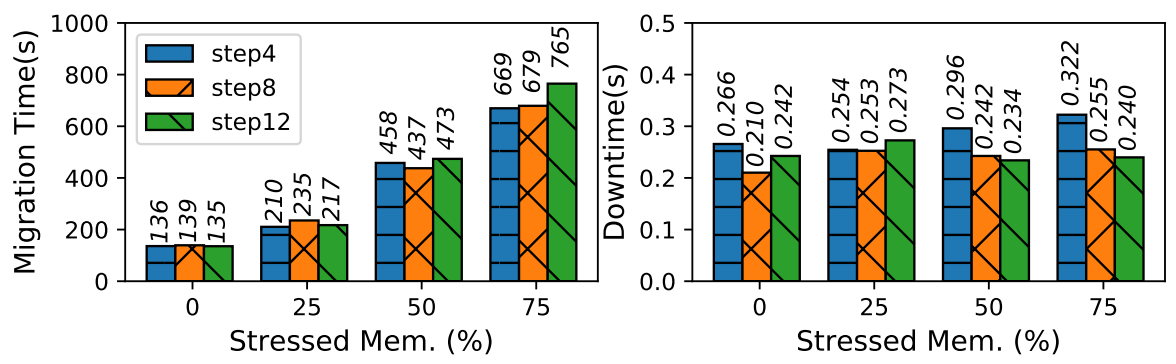

(a) live_migration_downtime_steps
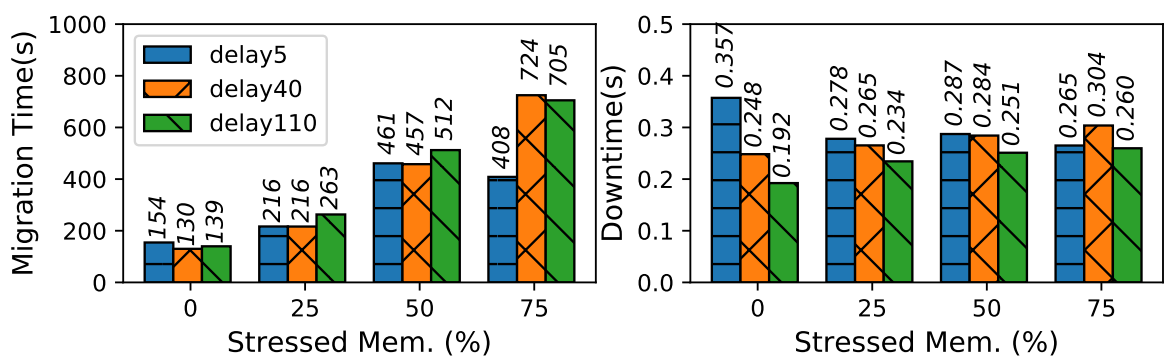

(b) live_migration_downtime_delay

Figure 6: Live migrations based on different step and delay settings

step8 $(500,8,75)$ leads to a better result in migration time compared to step12 and in downtime compared to step4 when VM memory is 75 percent stressed. We also notice that the setting $(500,10,5)$ is a better choice when VM has high dirty page rate and $(500,4,75)$ is better when the rate gets lower. When the dirty page rate is high, the migration time gets benefits from quickly raised downtime threshold while the downtime remains at a stable range. When it is low, the downtime gets benefits from smaller downtime threshold with slow adjustment. We should dynamically configure the optimal downtime setting tuple to improve both migration time and downtime based on the migration model for every live migration task.

\subsection{Live VM Migration in Parallel}

The default NOVA configuration of max allowed parallel migration is max_concurrent_live_migrations $=1$, which means only one live migration could be per- 

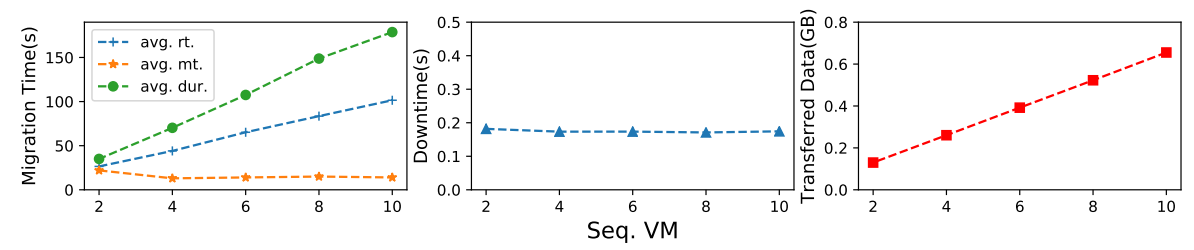

(a)
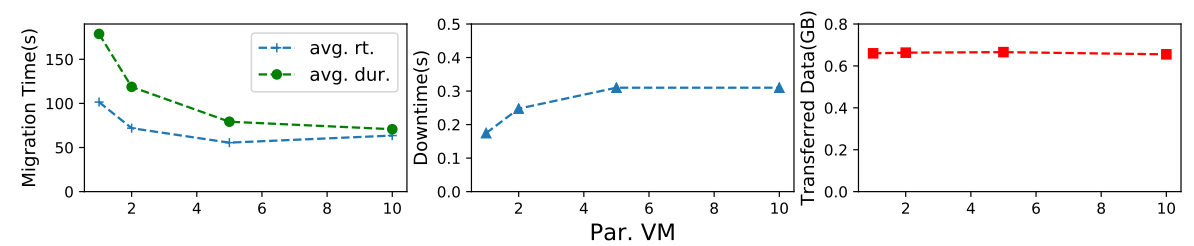

(b)

Figure 7: (a) Sequential migrations with different number of VMs; and (b) Multiple live migrations of $10 \mathrm{VMs}$ where $\mathrm{x}$-axis indicates the max allowed concurrent migration.

formed at the same time. In this experiment, we evaluate the migration duration

of one compute host that needs to evacuate all VMs to another. First, we need to change the default max allowed parallel migration to perform maximum $m$ live migrations in parallel. The CirrOS instances with tiny flavor are migrated between node compute2 and compute3. All migration operations are released at the same time with different maximum parallel migration. We measure the response time of each migration task and the total evacuation time of 10 idle CirrOS VMs. We also exam the sequential live migration with several VMs from 2 to 10.

Figure $7 \mathrm{a}$ indicates that the response time (rt), migration time (mt) and evacuation duration (dur) of sequential live migrations increase linearly with the number of VMs. Figure $7 \mathrm{~b}$ only demonstrates the rt and dur, as the mt equals to the rt in this parallel migration experiments. However, the parallel migrations could significantly reduce the total evacuation time and each migration time of 10 idle VMs. With the max allowed concurrent migration increasing from 1 to 10 , the total live migration evacuation time decreases by $59.6 \%$. Meanwhile, the 
migration time of each VM decreases up to $50 \%$.

As shown in Equation (19), 20], when $\Delta_{\text {network }}<\Delta_{\text {workload, }}$, the pre- and post-migration overheads constitute a large portion of the total migration time, e.g., parallel migration of the tiny flavor CirrOS VMs with 100Mbps bandwidth (Fig. 7p). Therefore, several pre- and post-live-migration processes concurrently running on both hosts can reduce the total evacuation time (15) and average response time $(18)$ compared to the sequential live migrations $(12),(17)$. Therefore, when the multiple VM evacuation happens in the same network path, we need to decide the sequential and parallel live migration based on both network and computing aspects to achieve a better total migration time (duration).

\subsection{Network-Aware Live Migration}

As the networking resources are limited, we pinpoint the essential network aspects that influence the efficiency of block live migration in SDN-enabled cloud computing, such as, the available network bandwidth, network patterns, SDN flow scheduling algorithms.

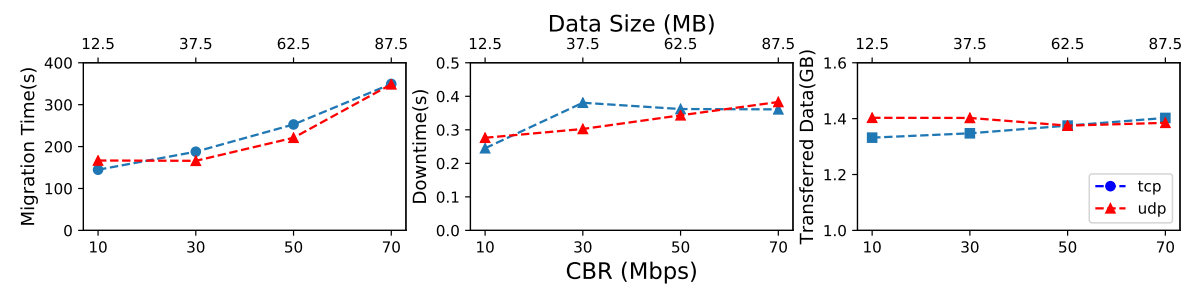

Figure 8: Block live migrations with TCP and UDP background traffic

TCP and UDP Traffic: Block live Migration is highly relative to the network bandwidth as well as the background traffic on the links. The total migration time and downtime are negatively correlated with the network bandwidth. Therefore, we measure the migration performance under the default downtime configuration with various network traffic scenarios with different constant bandwidth rate $(\mathrm{CBR})$ in TCP and burst transmission in UDP. UDP datagrams are sent in the same data size in every 10 seconds. The iperf3 [35] is used to generate background traffic between live migration source and 
destination hosts through the same path in SDN-enabled data center network. The image of VM is Ubuntu-16.04 with micro flavor under no stressed memory. Figure 8 indicates that, when the dirty page rate is 0 , the transferred data is not linearly increased with the migration time. The migration time is increased linearly with the bandwidth decreasing.

Dynamic SDN Flow Scheduling: In this experiment, we pinpoint the impact of the flow scheduling algorithm update rate on block live migration in SDN-enabled cloud computing. When SDN controller is proactively scheduling the flows, latencies exist between controller and switches (PacketOut message send to the switches and PacketIn to the controller). Moreover, in the flow tables, latencies occur when installing, deleting flow entities. The scheduler based on SDN controller (OpenDayLight) REST APIs proactively pushes the end-to-end flow in a certain time period to dynamically set the best path. The idle Ubuntu-16.04 instance with micro flavor is migrated from compute3 to compute9. As shown in Fig. 4, there are two shortest paths between compute3 and compute 9 that each one contains 5 OpenFlow nodes (OpenFlow-enabled switches). A round-robin scheduler rescheduling the traffic of live migration periodically based on these paths. We also use iperf3 to generate TCP and UDP traffic to evaluate the latency, TCP window size, and packet loss rate.

Figure $9 \mathrm{a}$ shows that the migration time is positively correlated with the update rate while the transferred data is just slightly increased. As the dynamic scheduling update rate increases, the link bandwidth rapidly decreases which leads to a large migration time. Meanwhile, Figure $9 \mathrm{~b}$ indicates that the TCP throughput goes down more frequently with high flow update rate. The TCP congestion window size decreases to 1.41 KBytes when the bandwidth is 0 bits/sec. Figure 10 shows the TCP and UDP protocol performance with different update rates from $0.1 \mathrm{~Hz}$ to $10 \mathrm{~Hz}$. The packet loss rate increase linearly with the update rate and the average maximum TCP latency (Round-Trip Time) is 2 times larger at $2 \mathrm{~Hz}$ than the minimum value at $0.1 \mathrm{~Hz}$. When the TCP traffic suffers the bandwidth degradation, the UDP transmission rate is always around $90 \mathrm{Mbps}$ regardless of the scheduling update rate. 


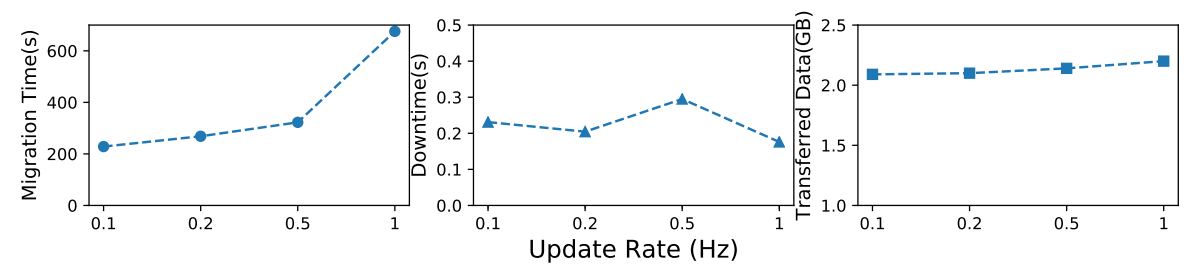

(a) Comparison of migrations

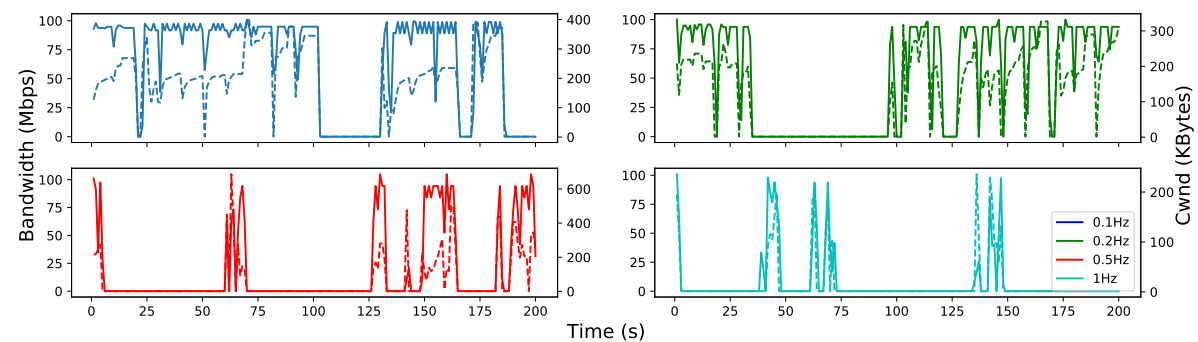

(b) Comparison of Iperf throughput: solid line indicated the Bandwidth and dot line indicated the Cwnd

Figure 9: Live migrations based on different SDN scheduling update rate

With the high flow entries updating in OpenFlow-enabled switches, the latencies between SDN controller and switches, and inside the switch flow tables have a significant influence on traffic forwarding performance. The network congestion leads to the high packet loss rate. The period of no traffic interval is caused by the TCP congestion avoidance algorithm. It decreases the data transfer rate when encounters packet loss based on the assumption that the loss due to the high latency and network congestion. Furthermore, the flow update rate could also impact the TCP window size that causes the bandwidth jitters due to the TCP slow start. In a highly dynamic network, the available bandwidth and delays in the routing paths can change frequently. Therefore, it is essential that optimize the update rate and best path selection of SDN forwarding scheduler based on the trade-off between OpenFlow-enabled switches performance (bandwidth degradation due to delays inside switches and between controller and switches) and the available network bandwidths and delays. 


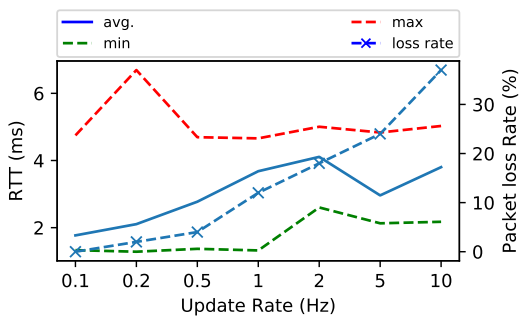

(a) TCP latency and packet loss rate

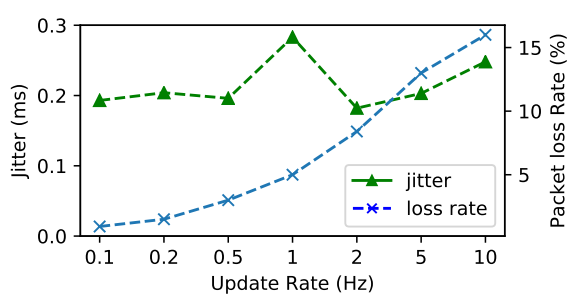

(b) UDP jitter and packet loss rate

Figure 10: Network performance with different SDN scheduling update rate

Table 3: Request response time without VM migration

\begin{tabular}{cccccc}
\hline Exp. & Duration(s) & RT(ms) & HTTP0 & HTTP200 & Total \\
\hline Initial & 1200 & 74.21 & 35 & 42310 & 51634 \\
Initial & 500 & 75.90 & 22 & 17435 & 21438 \\
Initial & 400 & 75.77 & 22 & 13893 & 17088 \\
scheduled & 400 & 65.380 & 17 & 14175 & 17357 \\
\hline
\end{tabular}

\subsection{Impacts on Multi-tier Application Response Time}

In this experiment, we evaluate the impact of VM live migration on the real

web application, such as MediaWiki, using WikiBench [36. It uses MediaWiki in the application server and real database dumps in the database server. In client VM, the wikijector as traffic injector controls the simulated client to reply the traces of real Wikipedia traffic. Regarding the scale of the testbed, we use 10 percent of Wikipedia trace to simulate the real traffic. The database and MediaWiki Apache servers are allocated in compute3, and one WikiBench injector as the client VM located in compute9. The client and server VMs are the Ubuntu instances with micro flavor and database server is with large flavor. The first scenario (c-93) is migrating the client VM to compute3 to simulate the consolidation (scheduled) to reduce the latency. The second one (s-39) is migrating the application server to compute 9 in order to evaluate the effect of live migration on application response time. 
Table 4: Application performance in 400 seconds

\begin{tabular}{cccccc}
\hline Exp. & MT(s) & RT(ms) & HTTP0 & HTTP200 & Total \\
\hline c-93 & 248.34 & 84.201 & 20 & 14166 & 17348 \\
s-39 & N/A & 192.273 & 109 & 13410 & 16558 \\
\hline
\end{tabular}

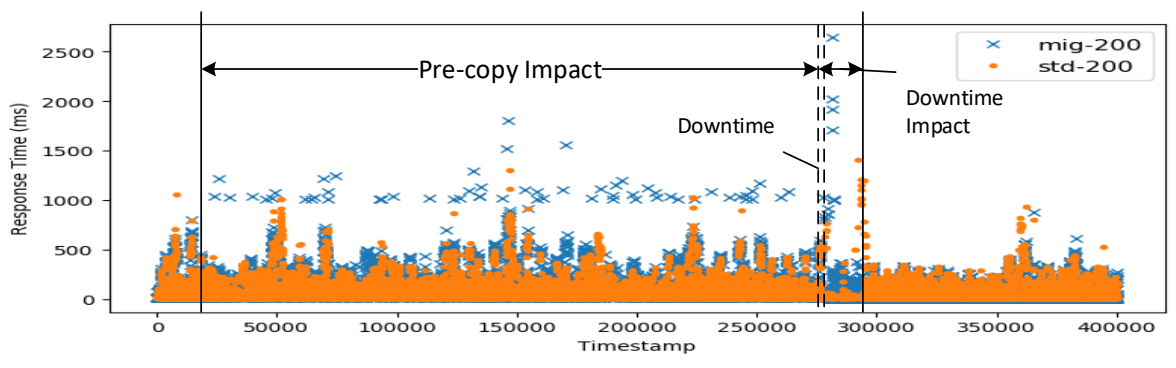

(a) Client VM migration

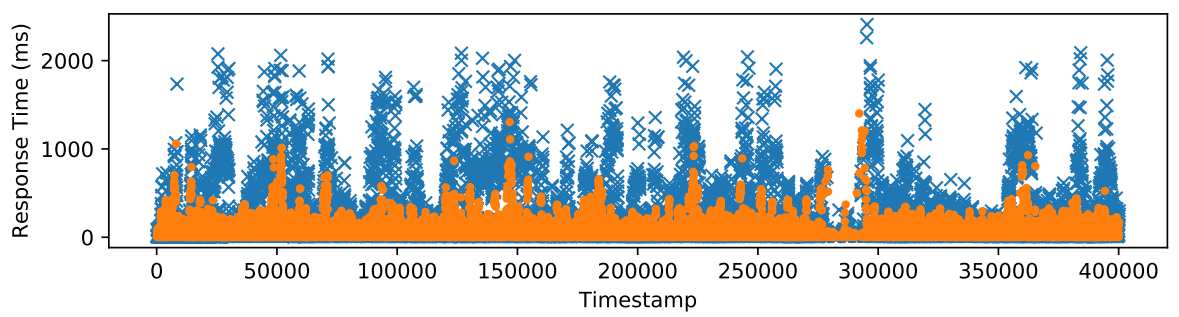

(b) Service VM migration segment

Figure 11: Response time of Wikipedia in 400s

In the scenario c-93, the major application traffic is outbound traffic from the destination host. Therefore, the live migration traffic would just slightly affect the QoS of web service. Table 3 indicates that the application response time (RT) is improved after the VM consolidation (scheduled). Figure 11 shows the initial response time (std-200) of the success requests (HTTP 200) and the response time of success requests during the client VM migration (mig200). It indicates that the response time is increased during the migration and the worst-case response time occurs after the downtime of client's live

VM migration because the application server needs to process extra requests 
Table 5: Server migration under different strategies

\begin{tabular}{ccccccc}
\hline Exp. & MT(s) & Duration(s) & RT(ms) & HTTP0 & HTTP200 & Total \\
\hline s-39 & N/A & 400 & 192.27 & 109 & 13410 & 16558 \\
AC & 908 & 1200 & 245.33 & 6722 & 18461 & 29915 \\
H-PC & 237 & 500 & 156.73 & 190 & 16906 & 20912 \\
\hline
\end{tabular}

and migration downtime postpones the response time of the requests which are sent before and during the downtime. On the other hand, if the injector and application server are located in the same host when the migration is performing, due to all requests happened inside the host, the live migration traffic will not affect the application response time.

However, in scenario s-39, i.e., the application traffic is sent to client VM (compute9), the pre-copy live migration traffic flow will contend for the shared bandwidth due to the same traffic direction. Therefore, the worst case response time may occur not only after downtime but during the migration time as shown in Fig. 11b. Meanwhile, Table 4 shows that the average response time of requests is dramatically larger than the migration of client VM. The request timeout (HTTP 0) happens much often due to the server migration.

We notice that the server migration from compute3 to compute 9 cannot finish in 20 minutes. For memory-intensive instances, like the Wikipedia server, there are two optional strategies to perform a successful live migration: Hybrid post-copy (H-PC) and Auto-convergence (AC). Thus, we evaluate the migration performance and impacts on the response time of the hybrid postcopy and auto-convergence strategies for Wikipedia server in the scenario s-39. Table 3 shows the initial response time of 1200 seconds, 500 seconds and 400 seconds time intervals without any migration as well as the average migration time (Duration), response time (RT), and the number of success (HTTP200), timeout (HTTP0), and total requests.

Hybrid post-copy: With the start of pre-copy mode, the post-copy migration will be activated if the memory copy iteration does not make at least 


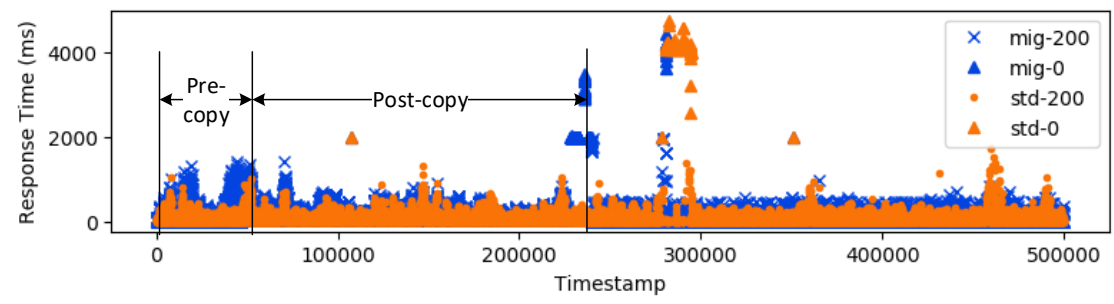

(a) Hybrid post-copy

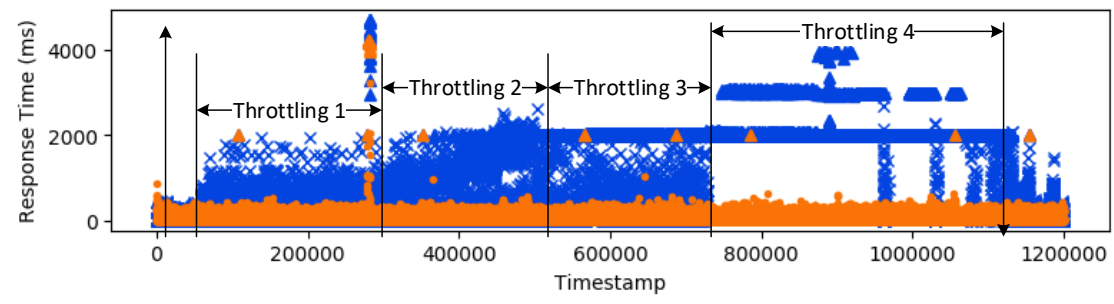

(b) Auto-convergence

Figure 12: Response time of successful server migrations

675 state on the source host. The VM will resume on the target host and fetch all missing pages as needed. However, the post-copy page fetching will slow down the VM which degrades the service performance and the VM will reboot if the network is unstable. The average response time of hybrid post-copy is better than the pre-copy migration as shown in Table 5 . The timeout requests are slightly increased during the post-copy migration. Furthermore, Figure 12 a indicates response time of success and timeout requests without migration (std200, std-0) and during the hybrid post-copy (mig-200, mig-0). It illustrates that under a stable network environment, the impacts of missing page fetching on

Auto-convergence: By throttling down the VM's virtual CPU, autoconvergence will only influence the workloads where the memory write speed 
is dependent on the CPU execution speed. As migration time flows it will continually increase the amount of CPU throttling until the dirty page rate is low enough for migration to finish. Figure $12 \mathrm{~b}$ indicates that the task of Wikipedia request has a worse response time under a larger throttling amount. The request tasks are highly related to the CPU execution speed. Therefore, the throttling down leads to a successful migration of the Wikipedia server. However, as the timeout threshold of a request is 2 seconds, the performance of the server is devastated under the last throttling down, i.e., most requests are timed out (mig-0). A larger timeout threshold for requests should be set according to the amount of throttling down. Although it can successfully perform the live server migration, the average response time is even larger than the precopy migration requests' (Table 5). Moreover, compare to the hybrid post-copy strategy, the auto-convergence leads to a much larger migration time.

For memory-intensive VMs, H-PC is a better strategy in a stable network environment. Otherwise, $\mathrm{AC}$ is the option for applications that dirty page rate is highly related to the CPU speed. Due to the throttling down, service time out should be increased accordingly.

\section{Conclusions and Future Work}

We established the mathematical model of block live migration to have a better understanding of the static downtime adjustment algorithm in OpenStack, as well as the parallel and sequential migration cost in the same network path. For the downtime adjustment algorithm, we should dynamically set the downtime configuration (maximum downtime, adjustment steps, and delays) to achieve the optimal migration performance. When non-network overheads, such as pre- and post-migration workloads, constitute a large portion of total migration time, parallel migration should be chosen to reduce the response time, downtime, and the total evacuation time of multiple migrations in the same path. We also evaluated the impacts of SDN scheduling update rate on live migration performance. The result suggests that a high update rate leads 
to a large TCP/UDP packet loss which will affect the migration performance.

From the QoS perspective, we investigated the response time pattern of client and server live migrations with pre-copy, hybrid post-copy, and autoconvergence strategies. For memory-intensive VM, as the pre-copy migration cannot finish in a reasonable time, we should choose hybrid post-copy to perform a successful migration if the network environment is stable. Otherwise, we could perform the auto-convergence feature during the pre-copy migration. However, the auto-convergence dramatically influences the application response time, i.e., requests are timed out because of the CPU slowdown. Moreover, for the precopy migration of server VM, as the migration and application traffic flows contend with each other, the worst-case response time will not just occur after the downtime but during the migration. Moreover, the models and parameters in our paper are compatible with other optimization technologies for single live VM migration [1, 3, 8, 9] or algorithms of multiple migrations [12, 14, 15, 16, 17, 18 because these work focus on different optimization factors. Therefore, the results in our paper still stand and can benefit other optimization methods and algorithms.

In the future, we plan to investigate the impact of these parameters' evaluation outcomes on the resource management in SDN-enabled cloud computing. In particular, we intend to investigate and develop: (a) the prediction model of live VM migration with static downtime adjustment algorithm and the optimal downtime adjustment configuration for different live migration tasks; (b) Deadline-aware multiple live VM migration planning by considering the parallel and sequential sequence in multiple and one network path; (c) SDN latencyaware traffic scheduling algorithm based on the trade-off between bandwidth increasing and rescheduling rate; and (d) QoS-aware resource scheduling strategy by considering application traffic pattern to minimize the influence of live migrations on application response time. 


\section{Acknowledgments}

This work is partially supported by an ARC Discovery Project [grant number DP160102414]; and the Chinese Scholarship Council.

\section{References}

[1] C. Clark, K. Fraser, S. Hand, J. G. Hansen, E. Jul, C. Limpach, I. Pratt, A. Warfield, Live migration of virtual machines, in: Proceedings of the 2nd Conference on Symposium on Networked Systems Design \& Implementation-Volume 2, USENIX Association, 2005, pp. 273-286.

[2] V. Mann, A. Gupta, P. Dutta, A. Vishnoi, P. Bhattacharya, R. Poddar, A. Iyer, Remedy: Network-aware steady state vm management for data centers, in: Proceedings of International Conference on Research in Networking, Springer, 2012, pp. 190-204.

[3] T. Guo, U. Sharma, P. Shenoy, T. Wood, S. Sahu, Cost-aware cloud bursting for enterprise applications, ACM Transactions on Internet Technology (TOIT) 13 (3) (2014) 10.

[4] J. Son, A. V. Dastjerdi, R. N. Calheiros, R. Buyya, Sla-aware and energyefficient dynamic overbooking in sdn-based cloud data centers, IEEE Transactions on Sustainable Computing 2 (2) (2017) 76-89.

[5] M. R. Hines, U. Deshpande, K. Gopalan, Post-copy live migration of virtual machines, ACM SIGOPS operating systems review 43 (3) (2009) 14-26.

765 [6] QEMU Project. Live block operation documentation, https: //kashyapc.fedorapeople.org/QEMU-Docs/_build/html/index.html, 2017 (accessed 11 Feb 2018).

[7] A. Mashtizadeh, E. Celebi, T. Garfinkel, M. Cai, et al., The design and evolution of live storage migration in vmware esx, in: Usenix Atc, Vol. 11, 2011, pp. 1-14. 
[8] A. Shribman, B. Hudzia, Pre-copy and post-copy vm live migration for memory intensive applications, in: Proceedings of European Conference on Parallel Processing, Springer, 2012, pp. 539-547.

[9] X. Song, J. Shi, R. Liu, J. Yang, H. Chen, Parallelizing live migration of virtual machines, ACM SIGPLAN Notices 48 (7) (2013) 85-96.

[10] S. Akoush, R. Sohan, A. Rice, A. W. Moore, A. Hopper, Predicting the performance of virtual machine migration, in: Proceedings of 2010 IEEE International Symposium on Modeling, Analysis \& Simulation of Computer and Telecommunication Systems (MASCOTS), IEEE, 2010, pp. 37-46.

[11] Z. Li, G. Wu, Optimizing vm live migration strategy based on migration time cost modeling, in: Proceedings of the 2016 Symposium on Architectures for Networking and Communications Systems, ACM, 2016, pp. 99-109.

[12] F. Xu, F. Liu, L. Liu, H. Jin, B. Li, B. Li, iaware: Making live migration of virtual machines interference-aware in the cloud, IEEE Transaction on Computers 63 (12) (2014) 3012-3025.

[13] M. Forsman, A. Glad, L. Lundberg, D. Ilie, Algorithms for automated live migration of virtual machines, Journal of Systems and Software 101 (2015) $110-126$.

[14] U. Deshpande, K. Keahey, Traffic-sensitive live migration of virtual machines, Future Generation Computer Systems 72 (2017) 118-128.

[15] S. Ghorbani, M. Caesar, Walk the line: consistent network updates with bandwidth guarantees, in: Proceedings of the first workshop on Hot topics in software defined networks, ACM, 2012, pp. 67-72.

[16] M. F. Bari, M. F. Zhani, Q. Zhang, R. Ahmed, R. Boutaba, Cqncr: Optimal vm migration planning in cloud data centers, in: Proceedings of Networking Conference, 2014 IFIP, IEEE, 2014, pp. 1-9. 
[17] G. Sun, D. Liao, V. Anand, D. Zhao, H. Yu, A new technique for efficient live migration of multiple virtual machines, Future Generation Computer Systems 55 (2016) 74-86.

[18] H. Wang, Y. Li, Y. Zhang, D. Jin, Virtual machine migration planning in software-defined networks., in: INFOCOM, IEEE, 2015, pp. 487-495. URL http://dblp.uni-trier.de/db/conf/infocom/infocom2015. html\#WangLZJ15

[19] W. Voorsluys, J. Broberg, S. Venugopal, R. Buyya, Cost of virtual machine live migration in clouds: A performance evaluation, in: Proceedings of IEEE International Conference on Cloud Computing, Springer, 2009, pp. $254-265$.

[20] S. Kikuchi, Y. Matsumoto, Impact of live migration on multi-tier application performance in clouds, in: Proceedings of 2012 IEEE 5th International Conference on Cloud Computing (CLOUD), IEEE, 2012, pp. 261-268.

[21] J. J. Herne, Auto-convergence feature, https://wiki.qemu.org/ Features/AutoconvergeLiveMigration, 2015 (accessed 11 Feb 2018).

[22] O. Sefraoui, M. Aissaoui, M. Eleuldj, Openstack: toward an open-source solution for cloud computing, International Journal of Computer Applications $55(3)$.

[23] Libvirt. Virtualization API, https://libvirt.org/, 2017 (accessed 25 Jan 2018).

[24] W. Hu, A. Hicks, L. Zhang, E. M. Dow, V. Soni, H. Jiang, R. Bull, J. N. Matthews, A quantitative study of virtual machine live migration, in: Proceedings of the 2013 ACM Cloud and Autonomic Computing Conference, ACM, 2013, p. 11.

[25] H. Liu, C.-Z. Xu, H. Jin, J. Gong, X. Liao, Performance and energy modeling for live migration of virtual machines, in: Proceedings of the 20th in- 
ternational symposium on High performance distributed computing, ACM, 2011, pp. 171-182.

[26] J. Son, R. Buyya, A taxonomy of software-defined networking ( $\operatorname{sdn})-$ enabled cloud computing, ACM Comput. Surv. 51 (3) (2018) 59:1-59:36. doi:10.1145/3190617 URL http://doi .acm .org/10.1145/3190617

[27] K. He, J. Khalid, A. Gember-Jacobson, S. Das, C. Prakash, A. Akella, L. E. Li, M. Thottan, Measuring control plane latency in sdn-enabled switches, in: Proceedings of the 1st ACM SIGCOMM Symposium on Software Defined Networking Research, ACM, 2015, p. 25.

[28] M. Kuźniar, P. Perešíni, D. Kostić, What you need to know about sdn flow tables, in: Proceedings of International Conference on Passive and Active Network Measurement, Springer, 2015, pp. 347-359.

[29] N. McKeown, T. Anderson, H. Balakrishnan, G. Parulkar, L. Peterson, J. Rexford, S. Shenker, J. Turner, Openflow: enabling innovation in campus networks, ACM SIGCOMM Computer Communication Review 38 (2) (2008) 69-74.

[30] Open vSwitch, https://www.openvswitch.org/, 2016 (accessed 15 Jan 2018).

[31] P. Svärd, B. Hudzia, J. Tordsson, E. Elmroth, Evaluation of delta compression techniques for efficient live migration of large virtual machines, ACM Sigplan Notices 46 (7) (2011) 111-120.

[32] A. N. Toosi, J. Son, R. Buyya, Clouds-pi: A low-cost raspberry-pi based micro data center for software-defined cloud computing, IEEE Cloud Computing 5 (5) (2018) 81-91.

850

[33] OpenDayLight Carbon release, https://docs.opendaylight.org/en/ stable-carbon/index.html, 2017 (accessed 25 Jan 2018). 
[34] C. King, Stress-ng, http://kernel.ubuntu.com/ cking/stress-ng/ 2018 (accessed 24 Feb 2018).

[35] Iperf3, https://iperf.fr/, 2016 (accessed 11 Feb 2018).

855 [36] E.-J. Van Baaren, Wikibench: A distributed, wikipedia based web application benchmark, Master's thesis, VU University Amsterdam (2009). 